\title{
THE CURRENT STATUS OF AUSTRIC \\ A review and evaluation of the lexical and morphosyntactic evidence
}

\author{
Lawrence A. Reid
}

\section{Introduction}

The purpose of this chapter is to review and evaluate the set of evidence that has so far appeared in support of a genetic relationship for the Austric family of languages, here defined as constituting the AN family as its eastern branch and the AA languages as its western branch. It thereby excludes consideration of evidence which suggests that the Tai-Kadai family of languages might be included as part of the family and avoids the obfuscation that discussion of the Austro-Tai hypothesis has had on the basic question of the genetic relationship of AA and AN.

There have been a number of articles, beginning with Schmidt (1906) that have presented sets of corresponding lexical items purporting to establish a genetic relationship between AA and AN. Much of this work has been shown to be spurious, but Diffloth (1994) presents a number of what he terms 'lexical agreements' between the two families which he considers to be probable. Subsequent work by Hayes $(1997,1999)$ has introduced a considerable number of new equations into the arena especially in the area of so-called 'basic vocabulary' that need to be evaluated. Some of these have already appeared in earlier work, but are reintroduced to us by Hayes in his attempt to show that, although, as Diffloth (1994: 312) says, 'the lexical evidence is not impressive, it is undoubtedly there', especially in that area of the lexicon that counts most strongly towards the establishment of a genetic relationship.

Schmidt (1906) was also the first to draw attention to the striking morphological comparisons that exist between the two families. Reid $(1994,1999)$ expanded on this work and noted also certain syntactic characteristics, which along with the reconstructed morphology suggested an ergative structure for the parent of the two families.

The first part of the chapter will be a detailed evaluation of the basic vocabulary comparisons between PAA and PAN proposed by Hayes (1999), to determine to what degree they may be said to constitute a body of cognates, supported by 
the usual requirements of recurrent sound correspondences and reasonable semantic equivalence. Hayes does not specifically claim that the pairs of forms he cites are cognates (this term does not appear in his paper), he refers to them by the less strict label of 'lexical comparison', a term which allows for forms which may be similar, not only because they are cognate, but also because they may be borrowings from one group into the other, or they may be the result of universal phonological developments, or they may simply be similar by chance. In order for the comparanda to constitute true cognates, it is imperative that a clear set of recurrent sound correspondences be established between the two proto-languages, and that the forms being compared have reasonably similar semantics.

The second part of the chapter will summarise the morphological evidence that has been proposed as evidence for a genetic relationship between AA and AN, and will discuss some of the alternative hypotheses that have been proposed to account for this evidence.

\section{The lexical evidence for Austric}

An adequate evaluation of Hayes' comparisons should consist of at least three parts: (1) an evaluation of the status of his PAA reconstructions and the methodology that he used to establish them, (2) an evaluation of the PAN reconstructions used in the comparisons and (3) an evaluation of the phonological correspondences and semantic features that supposedly relate the forms.

\section{Hayes'PAA reconstructions}

It is unclear from Hayes' paper whether the forms that he cites as evidence for each of his reconstructions constitute the total sum of his available evidence. I suspect that they probably do not, and that the few forms that he cites are representative of a (much?) larger body of evidence for which there was no room in the publication. However, I must assume that the forms that he cites constitute the best evidence available for his reconstructions.

For his PAA reconstructions, Hayes claims that most phonemic correspondences between the lexical items cited 'are in fact regular, at least where the consonants are concerned' (1999: 7), although in few cases does he attempt to make explicit what those regular correspondences are. As a non-specialist in AA languages, I have had to take this statement at face value in order to make my evaluation of his comparisons with PAN, although I suspect that a good deal of ingenuity was required in some cases to actually make the correspondences work. Hayes is, however, careful to indicate the relative time-depth of his own reconstructions.

The best claim to PAA status are those forms that he claims have reflexes in both the eastern and the western branches of the family. Of the approximately 
150 forms that he reconstructs, 76 have proposed reflexes from both major branches of the family. Of the remaining reconstructions, 70 have proposed reflexes in more than one language in one or more of the EAA subfamilies. The remaining forms have reflexes in only one language, but Hayes claims that in these cases the comparisons are, in effect, too good to be ignored. I have relabeled his reconstructions that do not have a western AA reflex as PEAA, and consider that these have lower probative value than those that can justly be claimed to be PAA.

\section{The Austronesian reconstructions}

On the AN side, all of Hayes' comparisons are with reasonably well-established reconstructions. Hayes used the list of 200 basic vocabulary reconstructions for PMP, provided in Blust (1993), rather than with the smaller set that have been reconstructed for PAN. However, in evaluating the comparisons, I have chosen to compare Hayes' AA reconstructions with PAN rather than with the historically subsequent PMP, whenever an appropriate PAN form exists. The PAN reconstructions with which I made my comparison are those which are also summarised alongside the PMP reconstructions in Blust (1999). Of the 150 or so comparisons for which Hayes cites a PMP reconstruction, there are some 79 for which the PMP form is a continuation of a reconstructed PAN reconstruction. This set potentially has high probative value when compared with an AA reconstruction. The 52 forms which compare with a PAA reconstruction (marked with a single asterisk in Table 8.1) have the highest value, while the remaining 27 that compare with only a PEAA reconstruction (marked with a double asterisk in Table 8.1) are of lower value. However, given the possibility that the Munda languages may have split off prior to the split of the eastern branch from pre-AN, this set of lexical comparisons may take on greater significance. The full set of potentially comparable PAA (and PEAA) forms with presently reconstructed PAN forms is shown in Table 8.1.

It is apparent that in making his comparisons, Hayes has operated on the assumption that PA must have been a highly affixing language, with PAN inheriting many of the forms in their affixed state, while their corresponding PAA forms were inherited either as roots, or with different affixes. Table 8.2 gives a list of some of the PAN forms that have been reanalysed by Hayes as originally consisting of a root plus one or more affixes.

Table 8.3 shows many of the proposed PA affixes that Hayes implies are present in his AN reconstructions. Although some of these forms may indeed have been affixes in the putative parent of PAN (as they are in PAN and some of its daughter languages) and some of the PAN reconstructions may have been morphologically complex (e.g. *Si-kan 'fish', *ma-ka-Sepal 'thick', ${ }^{*} \mathrm{C}<$ in $>$ aqi 'guts', even * $\mathrm{q}<\mathrm{al}>$ ejaw 'day', on the basis of comparisons such as Bontok ?algew 'sun, day', reflecting the full, infixed form, with reflexes of apparently unaffixed ma?égew 'to be fine, after rain', ?ag?agew 'morning', maggew 'handsome [bright appearance?]', etc.), it is methodologically unwise to equate any 
Table 8.1 Proposed lexical correspondences between PAA (and PEAA) and PAN (79)

\begin{tabular}{|c|c|c|c|}
\hline & $P A A$ and $P E A A$ & $P A N$ & Gloss \\
\hline 1.0 & Nature & & \\
\hline 1.1 & *qabuh & *qabu & ashes \\
\hline 1.2 & ** $[\mathrm{j}](\mathrm{a}) \mathrm{raw}$ & *qalejaw & day \\
\hline 1.3 & ${ }^{*}[\mathrm{~s}] \mathrm{uy},{ }^{*}[\mathrm{sa}](\mathrm{m}) \mathrm{puy}(\mathrm{s})$ & *Sapuy & fire \\
\hline 1.4 & **law & *danaw & lake \\
\hline 1.5 & $* * b(i, a) ! a l$ & $*$ bulaN & moon \\
\hline 1.6 & $* * k a[\tilde{n} j] \mathrm{al}$ & *quzaN & rain \\
\hline 1.7 & *qa(m)puc(i) & *timus & salt \\
\hline 1.8 & $* * \operatorname{si}[\mathrm{y}] \mathrm{aq}, * * \operatorname{su}[\mathrm{y}] \mathrm{ak}$ & *qasiRa & salt \\
\hline 1.9 & **ay & *qenay & sand \\
\hline 1.10 & *(m)pal & *qebel & smoke \\
\hline 1.11 & ${ }^{*} \mathrm{t}[\mathrm{o}] \mathrm{q}(\mathrm{i})$ & *bituqen & star \\
\hline 1.12 & **tamuq & *batu & stone \\
\hline 1.13 & *'[?]om & *daNum & water \\
\hline 2.0 & Flora & & \\
\hline 2.1 & $* *(\mathrm{~m}) \mathrm{b}[\mathrm{o} P \mathrm{a}] \mathrm{q}$ & *buaq & fruit \\
\hline 2.2 & $* * k(i, a) h i(u q)$ & *kaSiw & wood \\
\hline 3.0 & Fauna & & \\
\hline 3.1 & $* \operatorname{cu}(\mathrm{q})$ & *asu & $\operatorname{dog}$ \\
\hline 3.2 & *teloR & *qiCeluR & egg \\
\hline 3.3 & $*(\mathrm{n}) \mathrm{qa}(\mathrm{q})$ & *Sikan & fish \\
\hline 3.4 & $* * k[0] t(i)$ & *kuCu & head louse \\
\hline 3.5 & ${ }^{*} \mathrm{~b}[\partial \mathrm{w}]$ & *labaw & rat \\
\hline 3.6 & ${ }^{*}[\mathrm{su}][\mathrm{I}] \mathrm{aR}$ & *SulaR & snake \\
\hline 4.0 & Anatomy & & \\
\hline 4.1 & $* * k o[d(i)]$ & *likud & back \\
\hline 4.2 & *taPal, *ti?al & *tiaN & belly \\
\hline 4.3 & *cinqay, *canqay & ${ }^{*}$ CuqelaN & bone \\
\hline 4.4 & $*^{*} n[s] u q$ & *susu & breast \\
\hline 4.5 & $* *(n)[q 1] \mathrm{en}$ & *Calina & ear \\
\hline 4.6 & ${ }^{*} \operatorname{mo}(\mathrm{n}) \operatorname{ta}(\mathrm{q})$ & *maCa & eye \\
\hline 4.7 & *say, *suy & * SimaR & fat/oil \\
\hline 4.8 & *[?]aqi, *laqi & *Cinaqi & guts \\
\hline 4.9 & $*(\mathrm{n}) \operatorname{lem}[\mathrm{a}]$ & *(qa)lima & hand \\
\hline 4.10 & $* *(n) q o l u(q)$ & *qulu & head \\
\hline 4.11 & $* * p[a] l e(q)$ & *qaCay & liver \\
\hline 4.12 & $* c(i, \partial) c i$ & *Sesi/isi & meat/flesh \\
\hline 4.13 & $*(\mathrm{n}) \mathrm{qe}[\mathrm{R}]$ & *liqeR & neck \\
\hline 4.14 & *(ba) $\operatorname{Ra}(\mathrm{q})$ & *qabaRa & shoulder \\
\hline 4.15 & $* *[\operatorname{taN}] \mathrm{Gep}$ & *nipen & tooth \\
\hline 5.0 & Kinship & & \\
\hline 5.1 & *(qa)ma(ma) & *t-ama & father \\
\hline 5.2 & ${ }^{*} \mathrm{a}[\mathrm{x}] \mathrm{i},{ }^{*} \mathrm{bu}[\mathrm{x}] \mathrm{i},{ }^{*} \mathrm{mpa}[\mathrm{x}] \mathrm{i}$ & *bahi & female/woman \\
\hline 5.3 & *(n)qalay & *ma-RuqaNay & male/man \\
\hline 5.4 & *(na)na & $*$ t-ina & mother \\
\hline 5.5 & $*(k a l) ə w u(q)$ & ${ }^{*} \mathrm{Cau}$ & person \\
\hline
\end{tabular}


Table 8.1 Continued

\begin{tabular}{|c|c|c|c|}
\hline & $P A A$ and $P E A A$ & $P A N$ & Gloss \\
\hline 6.0 & Cultural artifacts & & \\
\hline 6.1 & $* *[u] y a q, *(\operatorname{sun})$ yum $[\mathrm{aq}]$ & *Rumaq & house \\
\hline 6.2 & *(n)jam[u]s & *jajan & name \\
\hline 6.3 & $\{* * \operatorname{Rom}\}$ & * zaRum & needle \\
\hline 6.4 & *k(a,u)la & *zalan & road \\
\hline 6.5 & *tal & ${ }^{*}$ CaliS & rope \\
\hline 7.0 & Descriptives & & \\
\hline 7.1 & *(can)yaya (q) & *ma-Raya & big \\
\hline 7.2 & $* * \mathrm{qi}[\mathrm{R}] \mathrm{u}(\mathrm{q})$ & *ma-baqeRu & new \\
\hline 7.3 & $* * t i(n) q a s(i)$ & *ma-tuqas & old (people) \\
\hline 7.4 & *yok & *ma-buRuk & rotten \\
\hline 7.5 & ${ }^{*} \mathrm{~g}(\mathrm{i}, \mathrm{a}) \mathrm{haq}(\mathrm{i})$ & *ma-Siaq & shy/ashamed \\
\hline 7.6 & *(n)qa[1], *qampa[1] & *ma-kaSepal & thick \\
\hline 8.0 & Verbs & & \\
\hline 8.1 & $* * k(\mathrm{n}) \mathrm{k}[\mathrm{o}] \mathrm{t}$ & *ma-takut & afraid \\
\hline 8.2 & *(n)yat(i) & *kaRat & bite \\
\hline 8.3 & $*[q]$ uyu & *Siup & blow \\
\hline 8.4 & $* *[\mathrm{P}] \mathrm{us}(\mathrm{i}), * * \mathrm{t}[\mathrm{u}]$ nus & ${ }^{*} \mathrm{CuNuh}$ & burn \\
\hline 8.5 & *po[1] i & *beli & buy \\
\hline 8.6 & ** (u) laqi & *piliq & choose \\
\hline 8.7 & $* *$ tayaq, **tayak & *taRaq & cut (wood) \\
\hline 8.8 & $* *$ tak, **tek & *tektek & cut (wood) \\
\hline 8.9 & ${ }^{*}(\mathrm{n}) \mathrm{ka}[\mathrm{l}]$ & *kalih & dig up \\
\hline 8.10 & $*(\mathrm{~m}) \mathrm{pe}(\mathrm{qi})$ & *Sepi & dream \\
\hline 8.11 & $*(\mathrm{in}) \mathrm{ka}(\mathrm{q})$ & *kaen & eat \\
\hline 8.12 & $* *$ qoy, ${ }^{* *}[\mathrm{qa}] \mathrm{loy}$ & ${ }^{*}$ qaluR (?) & flow \\
\hline 8.13 & *(n) kam & *gemgem & hold (in fist) \\
\hline 8.14 & ${ }^{*}(\mathrm{n})[\mathrm{r}] \mathrm{op}, *(\mathrm{c}, \mathrm{s})[\mathrm{r}] \mathrm{op}$ & *qaNup & hunt \\
\hline 8.15 & *ntaw & *Cawa & laugh \\
\hline 8.16 & $* *(\mathrm{~s})[\mathrm{R}] \mathrm{ai}$ & *kita & see \\
\hline 8.17 & $* *(\mathrm{n})$ qiq & $*$ taSiq & sew \\
\hline 8.18 & $*[?] \mathrm{aq}$ & *panaq & shoot \\
\hline 8.19 & $*(n) z o y$ & *tuduR & sleep \\
\hline 8.20 & *(z)yey & $* \mathrm{diRi}$ & stand \\
\hline 8.21 & *kaḷaw, *kumlaw & *Cakaw & steal \\
\hline 8.22 & ${ }^{*} \mathrm{~s}[\mathrm{e}] \mathrm{p}, *(\tilde{\mathrm{n}}) \mathrm{c}[\mathrm{e}] \mathrm{p}$ & ${ }^{*}$ sepsep & suck \\
\hline 8.23 & $*[\mathrm{P}] \partial \mathrm{q}(\mathrm{i}),{ }^{*}$ bur[əq] & *baReq & swell \\
\hline 8.24 & *la(n)[?]oy & *Najuy & swim \\
\hline 8.25 & $* \operatorname{taq}$ & *utaq & vomit \\
\hline 8.26 & $\{* * m a[\mathrm{q}]\}$ & *qumah & work in fields \\
\hline 8.27 & (can)qap & *ma-Suab & yawn \\
\hline
\end{tabular}

non-corresponding set of phonemes with an affix, unless justification can be found for it in the daughter languages. Such forms have therefore been eliminated from the set of potential cognates. This is not a trivial concern. Ignoring nonagreeing segments by calling them affixes without justification, allows for the inclusion of almost any non-cognate form into the comparative set. 
Table 8.2 Morphological reanalysis of PAN reconstructions (implied in Hayes 2000) (26)

\begin{tabular}{|c|c|c|}
\hline PAN (Blust 1999) & Gloss & Hayes' Reanalysis \\
\hline *qalejaw & day & $* \mathrm{q}<$ al $>$ ejaw \\
\hline *Sapuy & fire & $* \mathrm{~S}<\mathrm{ap}>\mathrm{uy}, *$ Sa-puy \\
\hline *qasiRa & salt & *qa-siRa \\
\hline *bituqen & star & *bituq-en \\
\hline *daNum & water & $* \mathrm{~d}<\mathrm{aN}>\mathrm{um}$ \\
\hline *Sikan & fish & Si-ka-n \\
\hline *CuqelaN & bone & $* \mathrm{Cu}-\mathrm{q}<\mathrm{el}>\mathrm{aN}$ \\
\hline *Calina & ear & "Calin-a \\
\hline *SimaR & fat/oil & ${ }^{*} \mathrm{~S}<\mathrm{im}>\mathrm{aR}$ \\
\hline *Cinaqi & guts & ${ }^{*} \mathrm{C}<\mathrm{in}>\operatorname{aqi}(?)$ \\
\hline *(qa)lima & hand & $*$ (qa) $\lim -\mathrm{a}$ \\
\hline *nipen & tooth & *nip-en \\
\hline *Cau & person & $* \mathrm{Ca}-\mathrm{u}$ \\
\hline *Rumaq & house & ${ }^{*} \mathrm{R}<\mathrm{um}>\mathrm{aq},{ }^{*} \mathrm{Rum}-\mathrm{aq}$ \\
\hline *najan & name & *najamn \\
\hline *zalan & road & *zala-n \\
\hline *Calis & rope & *Cal-iS \\
\hline *ma-kaSepal & thick & *ka-Se-pal \\
\hline *Siup & blow & $*$ Siu-p \\
\hline *kalih & dig up & *kal-ih \\
\hline *kaen & eat & "ka-en \\
\hline *qaluR (?) & flow & $* \mathrm{q}<\mathrm{al}>\mathrm{uR}, * \mathrm{qa}-\mathrm{luR}$ \\
\hline *Cawa & laugh & *Caw-a \\
\hline *panaq & shoot & ${ }^{*} \mathrm{p}<\mathrm{an}>\mathrm{aq}$ \\
\hline$* \mathrm{diRi}$ & stand & $*$ diR-i \\
\hline *baReq & swell & $* \mathrm{~b}<\mathrm{aR}>\mathrm{eq}, * \mathrm{baR}-\mathrm{eq}$ \\
\hline
\end{tabular}

Table 8.3 Hayes' proposed PA affixes in PAN reconstructions

\begin{tabular}{ll}
\hline Infixes & -al-, -an-, -aN-, -ap-, -aR-, -um-, -im-, -in-, -el- \\
Prefixes & qa-, Si-, Cu-, ka-, Se- \\
Suffixes & -en, -n, -u, -iS, -a, -aq, -i \\
\hline
\end{tabular}

The remaining set of PAN comparable forms are given in Table 8.4.

After eliminating the proposed sets that contain a PAN form that Hayes implies is morphologically complex, there remain some 19 sets, shown in Table 8.5.

The remaining AN reconstructions that Hayes cites are PMP forms that can be grouped into three types: (1) those which continue a PAN form but which have undergone an irregular phonological change, such as metathesis (M); (2) those which constitute a lexical replacement of an earlier PAN form (L) or are an innovation alongside a PAN form than has undergone a semantic shift in PMP (I); and (3) those for which no PAN form has as yet been reconstructed (?). These forms are shown in Table 8.6. 
Table 8.4 Potential PAA-PAN comparisons (31)

\begin{tabular}{|c|c|c|c|}
\hline & $P A A$ & $P A N$ & Gloss \\
\hline 1.1 & *qabuh & *qabu & ashes \\
\hline 1.7 & *qa(m)puc(i) & *timus & salt \\
\hline 1.10 & *(m)pocl & *qebel & smoke \\
\hline 3.1 & $* \mathrm{cu}(\mathrm{q})$ & *asu & $\operatorname{dog}$ \\
\hline 3.2 & *teḷoR & ${ }^{*} \mathrm{qiCeluR}$ & egg \\
\hline 3.5 & $* \mathrm{~b}[\partial \mathrm{w}]$ & 水labaw & rat \\
\hline 3.6 & $*[\mathrm{su}][1] \mathrm{aR}$ & *SulaR & snake \\
\hline 4.2 & *ta?al, *ti?al & ${ }^{*}$ tiaN & belly \\
\hline 4.4 & $* \mathrm{n}[\mathrm{s}] \mathrm{uq}$ & $*^{*}$ susu & breast \\
\hline 4.6 & $* \operatorname{mo}(n) \operatorname{ta}(q)$ & *maCa & eye \\
\hline 4.12 & ${ }^{*} \mathrm{c}(\mathrm{i}, \partial) \mathrm{ci}$ & ${ }^{*}$ Sesi/isi & meat/flesh \\
\hline 4.13 & ${ }^{*}(\mathrm{n}) \mathrm{qe}[\mathrm{R}]$ & *liqeR & neck \\
\hline 4.14 & $*(\mathrm{ba}) \operatorname{Ra}(\mathrm{q})$ & *qabaRa & shoulder \\
\hline 5.1 & *(qa)ma(ma) & *t-ama & father \\
\hline 5.2 & ${ }^{*} \mathrm{a}[\mathrm{x}] \mathrm{i}, * \mathrm{bu}[\mathrm{x}] \mathrm{i},{ }^{*} \mathrm{mpa}[\mathrm{x}] \mathrm{i}$ & "bahi & female/woman \\
\hline 5.3 & *(n)qalay & *ma-RuqaNay & male/man \\
\hline 5.4 & *(na)na & $* \mathrm{t}$-ina & mother \\
\hline 7.1 & *(can)yaya(q) & *ma-Raya & big \\
\hline 7.4 & *jok & *ma-buRuk & rotten \\
\hline 7.5 & ${ }^{*} \mathrm{~g}(\mathrm{i}, \mathrm{a}) \mathrm{haq}(\mathrm{i})$ & *ma-Siaq & shy/ashamed \\
\hline 8.2 & $*(n)$ yat(i) & *kaRat & bite \\
\hline 8.5 & ${ }^{*}$ pe $[!] \mathrm{i}$ & *beli & buy \\
\hline 8.10 & *(m)pe(qi) & *Sepi & dream \\
\hline 8.13 & $*(n)$ kəm & *gemgem & hold (in fist) \\
\hline 8.14 & ${ }^{*}(\mathrm{n})[\mathrm{r}] \mathrm{op},{ }^{*}(\mathrm{c}, \mathrm{s})[\mathrm{r}] \mathrm{op}$ & *qaNup & hunt \\
\hline 8.19 & *(n)zoy & *tuduR & sleep \\
\hline 8.21 & *kaḷaw, *kumlaw & *Cakaw & steal \\
\hline 8.22 & ${ }^{*} \mathrm{~s}[\mathrm{e}] \mathrm{p},{ }^{*}(\tilde{\mathrm{n}}) \mathrm{c}[\mathrm{e}] \mathrm{p}$ & *sepsep & suck \\
\hline 8.24 & *la(n)[?]oy & *Nayuy & swim \\
\hline 8.25 & $* \operatorname{taq}$ & *utaq & vomit \\
\hline 8.27 & $*$ (can) qap & *ma-huab & yawn \\
\hline
\end{tabular}

Of these groups, I have only included those marked with (?) in Table 8.6 as possible PMP comparisons, in that they may constitute a continuation of a PAN form which no longer exists in Formosan languages. These forms are considered to have lower probative value than true PAN reconstructions, and are labeled as PMP. From this set I have likewise eliminated those that Hayes implies were retentions of morphologically complex PA forms. The remaining set is provided in Table 8.7.

The final group of potentially comparable sets, and those that are of least value to supporting an Austric hypothesis are those that compare a PEAA form with a PMP form. This set (minus those that Hayes implies were retentions of morphologically complex PA forms) is provided in Table 8.8.

Sagart (email comm. 2001) has suggested 'some of the evidence for Austric is also extra-Formosan rather than PAN, suggesting again an early contact relationship rather than a genetic one'. If in fact there was a post-PAN return to the 
Table 8.5 Potential PEAA-PAN comparisons (19)

\begin{tabular}{|c|c|c|c|}
\hline & $P E A A$ & $P A N$ & Gloss \\
\hline 1.5 & $* * b(i, a) ! a l$ & *bulaN & moon \\
\hline 1.6 & ${ }^{*} k_{k} \mathrm{ka}[\tilde{\mathrm{n}} \mathrm{j}] \mathrm{al}$ & *quzaN & rain \\
\hline 1.9 & ***lay & *qenay & sand \\
\hline 1.12 & $* *$ tamuq & *batu & stone \\
\hline 2.1 & $* * \mathrm{k}(\mathrm{m}) \mathrm{b}[\mathrm{o}$ ?a] $\mathrm{q}$ & *buaq & fruit \\
\hline 2.2 & $* * k(i, a) h i(u p)$ & *kaSiw & wood \\
\hline 3.4 & $* * k[o] t(i)$ & ${ }^{*} \mathrm{kuCv}$ & head louse \\
\hline 4.1 & ${ }^{*} k_{k} k o[d(i)]$ & *likud & back \\
\hline 4.10 & *** (n)qolu(q) & *qulu & head \\
\hline 4.11 & **p[a]le(q) & *qaCay & liver \\
\hline 7.2 & $* * \mathrm{q}[\mathrm{R}] \mathrm{u}(\mathrm{q})$ & *ma-baqeRu & new \\
\hline 7.3 & $* * \operatorname{ti}(\mathrm{n}) \mathrm{qas}(\mathrm{i})$ & *ma-tuqas & old (people) \\
\hline 8.1 & $* *(\mathrm{n}) \mathrm{k}[\mathrm{o}] \mathrm{t}$ & *ma-takut & afraid \\
\hline 8.4 & 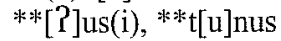 & ${ }^{*} \mathrm{CuNuh}$ & burn \\
\hline 8.6 & $* *$ (u)laqi & *piliq & choose \\
\hline 8.7 & **tayaq, **tayak & *taRaq & cut (wood) \\
\hline 8.8 & $* *$ tak, **tek & *tektek & cut (wood) \\
\hline 8.2 & $* *(\mathrm{~s})[\mathrm{R}] \mathrm{ai}$ & *kita & see \\
\hline 8.2 & $* *(\mathrm{n}) \mathrm{qiq}$ & ${ }^{*} \operatorname{taSiq}$ & sew \\
\hline
\end{tabular}

mainland, it is possible that some of the lexical sets proposed by Hayes which do not have a PAN reconstruction may be evidence for that. However, even these sets need to be critically evaluated, and are beyond the scope of this chapter.

\section{The sound correspondences}

My procedure was to begin with the reconstructed consonant system of PAN and PMP (Blust 1999: 34) shown in Table 8.9, and to compare, in order, each phoneme with the apparently corresponding phoneme in each of Hayes' comparisons.

It should be noted that I made no attempt to compare the vocalic systems. Although Hayes has made a provisional reconstruction of a 6-vowel system for PAA, he notes that 'additional vowel phonemes and diphthongs will probably have to be reconstructed eventually'. Comparison was based on the distributional features of the PAN phonemes in each of the (usually) disyllabic AN reconstructions. In many cases (100/154) the comparable form in Hayes was also disyllabic, and the determination of the appropriately corresponding phoneme was not difficult. The remaining forms, however, needed to be compared with what Hayes has reconstructed on the AA side with a monosyllabic form. For some 100 forms, the correspondence is between the final syllable of a PAN form (or the putative monosyllabic root of those forms that Hayes believes are continuations of affixed PA forms) and a PAA monosyllable. The full set of correspondences are shown in Table 8.10. Shading indicates distinctly different AA correspondences for the same PAN proto-phoneme. 
Table 8.6 Hayes' PAA-PMP comparisons (36)

\begin{tabular}{|c|c|c|c|}
\hline & $P A A$ & $P M P$ & Gloss \\
\hline 1.0 & Nature & & \\
\hline 1.1 & $* \mathrm{x}[\mathrm{a}] \mathrm{mb}[\mathrm{o}] 1$ & *Rabun (L) & cloud \\
\hline 1.2 & *buk & *qabuk (?) & dust \\
\hline 1.3 & *teq & ${ }^{*}$ taneq $(\mathrm{I})$ & earth, soil \\
\hline 1.4 & $*(\mathrm{~m})$ put & "kabut (?) & fog, mist \\
\hline 1.7 & *(bi)lat(i) & *kilat (M) & lightning \\
\hline 1.9 & *ger & *gurgur (L) & thunder \\
\hline 2.0 & Flora & & \\
\hline 2.2 & ${ }^{*}(\mathrm{~m})$ pun & *buna (?) & flower \\
\hline 2.3 & $*(\mathrm{n}) \mathrm{je}$ & *baliji (L) & grass \\
\hline 2.5 & $*(\mathrm{~s}) \mathrm{uy}(\mathrm{at})$ & "uRat (L) & root \\
\hline 2.6 & *(n)qay & *wakaR (L) & root \\
\hline 4.0 & Anatomy & & \\
\hline 4.2 & *(n)suk & *:buhek (M) & head hair \\
\hline 4.4 & *(di)!aq(i) & $* \operatorname{dilaq}(\mathrm{I})$ & tongue \\
\hline 5.0 & Kinship & & \\
\hline 5.1 & *(n)quP̂an[ak] & *anak (?) & child \\
\hline 5.2 & "saw $[\mathrm{a}]$ & *qasawa (?) & spouse \\
\hline 5.4 & *(lan)qe(q) & *laki(I) & male/man \\
\hline 6.0 & Cultural artifacts & & \\
\hline 6.1 & $*(\mathrm{n}) \mathrm{t}$ to & ${ }^{*}$ qatep (?) & roof/thatch \\
\hline 7.0 & Descriptives & & \\
\hline 7.2 & *(i)tem & ${ }^{*}$ ma-qiitem (L) & black \\
\hline 7.3 & *(z)len & *ma-dindin (?) & cold \\
\hline 7.6 & *p(a,u)yan & *ma-Ranaw (L) & dry \\
\hline 7.8 & *jar[?]uq & *ma-zauq (L) & far \\
\hline 7.11 & *(n)kit & *kepit (?) & narrow \\
\hline 7.12 & ${ }^{*} \mathrm{\gamma a}(\mathrm{k}, \mathrm{q})$ & ${ }^{*}$ ma-iRaq (L) & red \\
\hline 7.13 & ${ }^{*} \mathrm{su}(\mathrm{q})$ & *ma-busuk (I) & rotten \\
\hline 7.14 & *(n)zekiq & *dikiq (I) & small \\
\hline 8.1 & ${ }^{*} r(a, u) w a(i)$ & *mañawa (?) & breathe \\
\hline 8.0 & Verbs & & \\
\hline 8.2 & ${ }^{*} \operatorname{maq}(\mathrm{i})$ & ${ }^{*} \operatorname{mamaq}(\mathrm{L})$ & chew \\
\hline 8.4 & *[?]ay & *maRi (?) & come \\
\hline 8.5 & *[?]om & *inum (?) & drink \\
\hline 8.6 & $* t(a, u)(m) p u q$ & *nabuq (?) & fall \\
\hline 8.7 & $* \mathrm{~m}[\mathrm{~b}] \mathrm{uk}$ & *tu(m)buq (?) & grow \\
\hline 8.8 & $\left.{ }^{*} z \partial 1\right][\partial y(\mathrm{i})]$ & *dejeR (L) & hear \\
\hline 8.15 & *(n)qay $(\mathrm{i})$ & *kaRi (?) & say \\
\hline 8.21 & * yot(s) & ${ }^{*}$ peRes (I) & squeeze \\
\hline 8.20 & *(ba)!̣a(i) & *belaq (?) & split \\
\hline 8.22 & (n) $\operatorname{cuk}(\mathrm{i})$ & "suksuk (?) & stab \\
\hline 8.23 & $* \mathrm{da}(\mathrm{q})$ & *tudaq(?) & throw \\
\hline
\end{tabular}


Table 8.7 Potential PAA-PMP comparisons (13)

\begin{tabular}{|c|c|c|c|}
\hline & $P A A$ & $P M P$ & Gloss \\
\hline 1.2 & "kbuk & *qabuk & dust \\
\hline 1.4 & ${ }^{*}(\mathrm{~m}) \mathrm{put}$ & *kabut & fog, mist \\
\hline 5.1 & *(n)quPan[ak] & *anak & child \\
\hline 5.2 & *saw $[a]$ & * qasawa & spouse \\
\hline 6.1 & $*(n)$ top & *qatep & roof/thatch \\
\hline 7.3 & $*(z)$ len & *ma-dindin & cold \\
\hline 7.7 & $*$ tu[l] & *pundul & dull/blunt \\
\hline 8.1 & ${ }^{*} \mathrm{r}(\mathrm{a}, \mathrm{u}) \mathrm{wa}(\mathrm{i})$ & *mañawa & breathe \\
\hline 8.6 & ${ }^{*} t(a, u)(m) p u q$ & *nabuq & fall \\
\hline 8.7 & ${ }^{*} \mathrm{~m}[\mathrm{~b}] \mathrm{uk}$ & $* t u(m) b u q$ & grow \\
\hline 8.20 & *(ba)laq(i) & *belaq & split \\
\hline 8.22 & ${ }^{*}(\mathrm{n}) \operatorname{cuk}(\mathrm{i})$ & *suksuk & stab \\
\hline 8.23 & $* \mathrm{da}(\mathrm{q})$ & *tudaq & throw \\
\hline
\end{tabular}

Table 8.8 Potential PEAA-PMP comparisons (13)

\begin{tabular}{|c|c|c|c|}
\hline & $P E A A$ & $P M P$ & Gloss \\
\hline 4.3 & ${ }^{* * *}(\mathrm{~m}) \mathrm{paq}$ & *baqbaq & mouth \\
\hline 5.3 & $* * n a(q)$ & *bana & husband \\
\hline 7.4 & **bor, **bonər & *ma-bener & correct, true \\
\hline 7.5 & **maz & ${ }^{*}$ cemeD (?) & dirty \\
\hline 7.7 & $* * *_{t u}[1]$ & *pundul (?) & dull/blunt \\
\hline 7.9 & $* * \mathrm{i}[\mathrm{?}] \mathrm{ak},{ }^{*} \mathrm{k}_{\mathrm{u}} \mathrm{u}[\mathrm{?}] \mathrm{aq}$ & *ma-pia & good \\
\hline 7.10 & **abaRe(n)qot & *ma-beReqat & heavy \\
\hline 7.15 & **; & *ma-baseq (?) & wet \\
\hline 8.11 & $* *$ (i)lop & *qinep & lie down \\
\hline 8.14 & **ntuk & *tuktuk & pound \\
\hline 8.18 & **zaq(i),**ñjaqi & *luzaq & spit \\
\hline 8.19 & $* * \mathrm{ta}[\mathrm{q}]$ & *sitaq & split \\
\hline 8.24 & *** (n)kət & *hiket & tie \\
\hline
\end{tabular}

Table 8.9 PAN and PMP phonemic systems

\begin{tabular}{|c|c|c|c|c|c|c|c|c|c|c|c|c|}
\hline \multicolumn{7}{|c|}{$P A N$} & \multicolumn{6}{|c|}{$P M P$} \\
\hline $\mathrm{p}$ & $\begin{array}{l}\mathrm{t} \\
\mathrm{C}\end{array}$ & $\mathrm{c}$ & & $\mathrm{k}$ & $q$ & & $\mathrm{p}$ & $\mathrm{t}$ & $c$ & & $\mathrm{k}$ & $q$ \\
\hline b & $\mathrm{d}$ & z & $\mathrm{j}$ & $\mathrm{g}$ & & & $\mathrm{b}$ & $\mathrm{d}$ & $\mathrm{z}$ & $\mathrm{j}$ & $\mathrm{g}$ & \\
\hline $\mathrm{m}$ & $\stackrel{\mathrm{n}}{\mathrm{N}}$ & $\tilde{\mathrm{n}}$ & & $\eta$ & & & $\mathrm{m}$ & $\mathrm{n}$ & $\tilde{n}$ & & n] & \\
\hline & $\begin{array}{l}\mathrm{S} \\
1\end{array}$ & $\mathrm{~s}$ & & & & $\mathrm{~h}$ & & $\begin{array}{l}\mathrm{s} \\
1\end{array}$ & & & & $\mathrm{~h}$ \\
\hline & $\mathrm{r}$ & & & $\mathrm{R}$ & & & & $\mathrm{r}$ & & & $\mathrm{R}$ & \\
\hline$w$ & & & $\mathrm{y}$ & & & & $w$ & & & & $y$ & \\
\hline
\end{tabular}


Table 8.10 AN-AA phonological correspondences (based on Hayes' complete set of proposed lexical correspondences)

\begin{tabular}{|c|c|c|c|c|}
\hline \multirow[t]{2}{*}{$P A N$} & \multirow[t]{2}{*}{$P M P$} & \multirow[t]{2}{*}{$P A A$} & \multicolumn{2}{|l|}{ Reconstruction \# } \\
\hline & & & $P A N$ & $P M P$ \\
\hline \multirow{5}{*}{$\begin{array}{l}\text { pV.CV } \\
\text {-p- }\end{array}$} & pV.CV & $\varnothing$. & $8.6,8.18$ & $77,7.9,8.21$ \\
\hline & & $\begin{array}{l}-(m) p- \\
-m p-\end{array}$ & $\begin{array}{l}1.3 \\
(7.6)\end{array}$ & \\
\hline & $-p-$ & $(\mathrm{m}) \mathrm{p}-$ & 8.10 & 3.4 \\
\hline & & & 4.15 & \\
\hline & & $(n) q_{-}$ & $(7.6)$ & \\
\hline \multirow[t]{2}{*}{$-p$} & $-\mathbf{p}$ & $-p$ & 8.14 & $6.1,8.11$ \\
\hline & & 0 & 8.3 & \\
\hline CVp.CVp & & $\mathrm{CVp}$ & 8.16 & \\
\hline \multirow{4}{*}{ tV.CV } & tV.CV & $\varnothing$. & $8.1,8.17,4.2$ & $8.7,8.13,8.17,8.23$ \\
\hline & & tV.CV & $7.3,8.7,8.10$ & \\
\hline & & $t-V C$ & & 1.3 \\
\hline & & sV.CV & 1.7 & \\
\hline tVC.tVC & tVC.tVC & tVC & 8.8 & 8.14 \\
\hline \multirow[t]{2}{*}{$-t-$} & $-t-$ & & $1.10,1.11,8.25$ & $7.2,8.19$ \\
\hline & & {$[\mathrm{R}]$ - } & 8.17 & \\
\hline$-t$ & $-t$ & $-\mathrm{t}(\mathrm{i})$ & 8.2 & $\begin{array}{l}1.4,1.7,2.5,7.10,7.11 \\
8.16,8.24\end{array}$ \\
\hline \multirow[t]{6}{*}{$\mathrm{kV}, \mathrm{CV}$} & kV.CV & & 8.2 & 1.4 \\
\hline & & kV.CV & 2.2 & 3.4 \\
\hline & & & $3.4,4.1,8.21$ & \\
\hline & & $(\mathrm{n}) \mathrm{k}-$ & $8.9,8.11$ & \\
\hline & & qV.CV & 7.6 & \\
\hline & & (n) $q-$ & & $7.11,8.15$ \\
\hline \multirow[t]{5}{*}{$-k-$} & $-\mathrm{k}-$ & $-k-$ & & $7.14,8.3,8.26$ \\
\hline & & $-\mathrm{k}$ & & 8.12 \\
\hline & & $(n) \mathrm{k}-$ & 8.1 & 8.24 \\
\hline & & (n) $q-$ & 3.3 & $1.6,5.4$ \\
\hline & $-k$ & & & $\begin{array}{l}1.2,3.1,3.4,4.1,4.2, \\
76.87\end{array}$ \\
\hline \multirow{2}{*}{$-k$} & $-\mathbf{k}$ & $\begin{array}{l}-\mathrm{k} \\
-\mathrm{ki}\end{array}$ & 7.4 & $\begin{array}{l}1.10,8.1 \\
3.2\end{array}$ \\
\hline & & $-(q)$ & & 7.13 \\
\hline CVk.CVk & CVk.CVk & CVk & 8.8 & $8.14,8.22$ \\
\hline \multirow[t]{4}{*}{ qV.CV } & $q V . C V$ & $\varnothing$. & $\begin{array}{l}1.9,1.10,3.2 \\
(8.12), 8.14\end{array}$ & $\frac{1.2,1.8,5.2,6.1,7.2}{8.1}$ \\
\hline & & & $1.1,1.7,(8.12)$ & \\
\hline & & qV.CV & 4.10 & \\
\hline & & [i]a. & 1.2 & \\
\hline \multirow[t]{3}{*}{$-q-$} & $-q-$ & $\begin{array}{l}-q- \\
\text { (n)q- }\end{array}$ & $\begin{array}{l}1.11,4.3,4.8 \\
4.13\end{array}$ & $2.1,8.10,8.16,7.10$ \\
\hline & & $-q$ & & 7.1 \\
\hline & & & $2.1,7.5,8.6$ & $1.3,1.6,4.4,7.8,7.14$ \\
\hline
\end{tabular}


Table 8.10 Continued

\begin{tabular}{|c|c|c|c|c|}
\hline \multirow[t]{2}{*}{$P A N$} & \multirow[t]{2}{*}{$P M P$} & \multirow[t]{2}{*}{$P A A$} & \multicolumn{2}{|l|}{ Reconstruction \# } \\
\hline & & & $P A N$ & $P M P$ \\
\hline$-q$ & $-q$ & $\begin{array}{l}-q \\
-(q) \\
-1\end{array}$ & $\begin{array}{l}(8.7), 8.17,8.18 \\
(8.8)\end{array}$ & $\begin{array}{l}7.15,8.2,8.6,8.18,8.20 \\
7.12,8.23\end{array}$ \\
\hline $\mathrm{CVq} \cdot \mathrm{CVq}$ & $\mathrm{CVq} \cdot \mathrm{CVq}$ & $\mathrm{CVq}$ & $4.5,5.5,(8.4)$ & 4.3 \\
\hline CV.CV & & $\begin{array}{l}\text { O. } \\
\text { tV.CV } \\
\text { tVC } \\
\text { ntVC } \\
\text { CV.CV } \\
\text { cV.CV }\end{array}$ & $\begin{array}{l}8.21 \\
(8.4) \\
6.5 \\
8.15 \\
3.2 \\
4.3\end{array}$ & \\
\hline$-\mathrm{C}-$ & & $\begin{array}{l}-\mathrm{t}- \\
-\mathrm{C}- \\
-1-\end{array}$ & $\begin{array}{l}4.6 \\
3.4 \\
4.17\end{array}$ & \\
\hline cV.CV & & & $1.11,1.12,7.2$ & $7.5,3,4.2,5.3,7.13$ \\
\hline bV.CV & bV.CV & $\begin{array}{l}0 . \\
\text { bV.CV } \\
\text { (bV)- } \\
\text { b- } \\
\text { p- } \\
\text { (m) }- \text { - }\end{array}$ & $\begin{array}{l}7.4 \\
1.5,(5.2), 8.23 \\
4.15 \\
2.1 \\
8.5\end{array}$ & $\begin{array}{l}(7.15), 8.9,8.25 \\
(7.4), 7.10,(7.15) \\
(7.16) \\
8.20 \\
(7.4),(7.16) \\
8.12 \\
2.2\end{array}$ \\
\hline bVC.bVC & & $(\mathrm{m}) \mathrm{p}-$ & $4.13,(5.2)$ & 4.3 \\
\hline$-b-$ & & $\begin{array}{l}-\mathrm{b}- \\
(\mathrm{m}) \mathrm{b}-\end{array}$ & $\begin{array}{l}1.1 \\
2.1\end{array}$ & \\
\hline & $-b-$ & $\begin{array}{l}-m b- \\
m[b]- \\
b- \\
\mathrm{p}_{-}^{-} \\
-\mathrm{mp}-\end{array}$ & 3.5 & $\begin{array}{l}1.1 \\
8.7 \\
1.2 \\
1.4 \\
8.6\end{array}$ \\
\hline $\begin{array}{l}-b \\
\text { dV.CV }\end{array}$ & dV.CV & $\mathrm{p}$ & $1.4,1.13$ & 8.27 \\
\hline & dVC.dVC & $\begin{array}{l}\text { dV.CV } \\
\text { (d)- } \\
\text { zV.CV } \\
\text { (z)- } \\
\text { (z) VC }\end{array}$ & 8.20 & $\begin{array}{l}2.4 \\
4.4 \\
2.1,7,14,8.8 \\
\\
7.3\end{array}$ \\
\hline$-d-$ & & d- & 8.19 & $8.17,8.23$ \\
\hline $\begin{array}{l}-n d- \\
-d\end{array}$ & & $\begin{array}{l}t- \\
-d\end{array}$ & $\begin{array}{l}4.1(?) \\
4.1(?)\end{array}$ & 77 \\
\hline$-\mathbf{j}-$ & & $(n) j^{-}$ & $\begin{array}{l}6.2 \\
1.2\end{array}$ & 2.3 \\
\hline g & & & & \\
\hline
\end{tabular}

(Table 8.10 continued) 
Table 8.10 Continued

\begin{tabular}{|c|c|c|c|c|}
\hline \multirow[t]{2}{*}{$P A N$} & \multirow[t]{2}{*}{$P M P$} & \multirow[t]{2}{*}{$P A A$} & \multicolumn{2}{|c|}{ Reconstruction \# } \\
\hline & & & $P A N$ & $P M P$ \\
\hline $\mathrm{zV} . \mathrm{CV}$ & $\mathrm{zV} . \mathrm{CV}$ & $\begin{array}{l}\varnothing . \\
j V . C V \\
n j- \\
\text { LVCV }\end{array}$ & 6.4 & $\begin{array}{l}7.8,816 \\
7.1\end{array}$ \\
\hline$-z-$ & $-z-$ & $\begin{array}{l}\mathrm{n} j \mathrm{j} \\
-[\tilde{\mathrm{n}} \mathrm{j}]-\end{array}$ & 1.6 & $(8.18)$ \\
\hline & & $z-$ & & $(8.18)$ \\
\hline & $\mathrm{mV} . \mathrm{CV}$ & 0. & & $3,1,8.4$ \\
\hline $\mathrm{mV} . \mathrm{CV}$ & & mV.CV & 4.16 & 4.1 \\
\hline$-\mathrm{m}-$ & $-m-$ & $\begin{array}{l}\mathrm{m}- \\
-\mathrm{m}- \\
-(\mathrm{m}) \mathrm{p}-\end{array}$ & $\begin{array}{l}3.2 \\
8.26,4.9,5.1 \\
6.1 \\
1.7\end{array}$ & $7.5,8.2$ \\
\hline$-m$ & $\tilde{\mathbf{n} V . C V}$ & $\begin{array}{l}-\mathrm{m} \\
\mathrm{rC} . \mathrm{CV}\end{array}$ & $1.13,6.3,8.13$ & $\begin{array}{l}7.2,8.5,8.13 \\
8.1\end{array}$ \\
\hline & NV.CV & tVCV & & 7.6 \\
\hline$-n-$ & $-n-$ & $\begin{array}{l}n- \\
-n- \\
-n \\
1-\end{array}$ & $1.4,1.9$ & $\begin{array}{l}5.3 \\
5.1,7.4 \\
8.9 \\
3.1,8.11\end{array}$ \\
\hline$-n$ & $-\mathbf{n}$ & $\frac{-1}{-m[u] S}$ & $\frac{1.1}{6.2}$ & $2.4,8.17$ \\
\hline gV.CV & & 0. & 6.1 & \\
\hline$-\mathbf{y}-$ & $-\mathbf{y}-$ & $\begin{array}{l}-1]- \\
-1\end{array}$ & 4.5 & $\begin{array}{l}8.8 \\
2.2,7.6\end{array}$ \\
\hline-9 & $-\eta$ & $-\eta$ & 4.3 & $7.3,8.25$ \\
\hline $\begin{array}{l}\text { NV.CV } \\
\text {-N- }\end{array}$ & & IV.CV & $\begin{array}{l}8.24 \\
5.3\end{array}$ & . \\
\hline & & $\begin{array}{l}-n- \\
(\mathrm{c}, \mathrm{s})[\mathrm{I}] \\
(\mathrm{n})[\mathrm{I}]\end{array}$ & $\begin{array}{l}8.4 \\
(8.14) \\
(8.14)\end{array}$ & \\
\hline$-\mathrm{N}$ & & 4 & 4.2 & \\
\hline SV.CV & & Ø. & $3.3,8.3,8.10$ & \\
\hline & & sV.CV & 3.6 & \\
\hline & & {$[\mathrm{s}] \mathrm{V} . \mathrm{CV}$} & $(1.3)$ & \\
\hline & & sVC & 4.7 & \\
\hline & & {$[\mathrm{s}] \mathrm{VC}$} & (1.3) & \\
\hline & & CVCV & 4.12 & \\
\hline & & gVCCV & 7.5 & \\
\hline$-S-$ & & h- & 2.2 & \\
\hline & & $9-$ & 8.17 & \\
\hline$-S$ & & $-s(i)$ & 7.3 & \\
\hline & sV.CV & $\varnothing$. & अ४१ & 8.19 \\
\hline sV.CV & & $\begin{array}{l}\text { sV.CV } \\
{[n] s V}\end{array}$ & 1.8 & 5.2 \\
\hline
\end{tabular}

(Table 8.10 continued) 
Table 8.10 Continued

\begin{tabular}{|c|c|c|c|c|}
\hline \multirow[t]{2}{*}{$P A N$} & \multirow[t]{2}{*}{$P M P$} & \multirow[t]{2}{*}{$P A A$} & \multicolumn{2}{|c|}{ Reconstruction \# } \\
\hline & & & $P A N$ & $P M P$ \\
\hline & & cV.CV & & 8.3 \\
\hline \multirow{3}{*}{ sVC.sVC } & & sVC & $(4.22)$ & \\
\hline & sVC.sVC & $\mathrm{cVC}$ & $(4.22)$ & 8.22 \\
\hline & $-s-$ & $s-$ & & 7.13 \\
\hline \multirow[t]{2}{*}{$-s-$} & & c- & 3.1 & 1.10 \\
\hline & & $-c-$ & 4.12 & 7.15 \\
\hline \multirow[t]{2}{*}{$-s$} & & $-c(i)$ & 1.7 & 1.5 \\
\hline & hV.CV & $\varnothing$. & & $8.24,8.27$ \\
\hline \multirow[t]{2}{*}{$-\mathfrak{h}-$} & & (n) $\mathrm{s}-$ & & 4.2 \\
\hline & $-\mathbf{h}-$ & $\begin{array}{l}{[\mathrm{q}]} \\
{[\mathrm{x}]}\end{array}$ & 5.2 & \\
\hline \multirow[t]{2}{*}{$-h$} & & $-s$ & 8.4 & \\
\hline & & [q] & 8.26 & \\
\hline \multirow[t]{2}{*}{ IV.CV } & IV.CV & $\emptyset$. & $3.5,4.1,4.13$ & $2.3,(3.3), 8.18$ \\
\hline & & lCV.CV & $\begin{array}{l}4.9,8.6 \\
1.5,3.2,3.6\end{array}$ & \\
\hline \multirow[t]{5}{*}{$-1-$} & $-1-$ & $-1-$ & $4.10,6.4$ & $1.7,(3.3)$ \\
\hline & & $-[1]-$ & 8.5 & $1.6,8.25$ \\
\hline & & -1 & 6.5 & \\
\hline & & $1-$ & 4.5 & $1.5,4.4,8.20$ \\
\hline & & {[] $1-$} & 8.12 & \\
\hline \multirow[t]{6}{*}{-1} & & -1 & 1.10 & \\
\hline & & $-[1]$ & $7.6,8.9$ & 7.7 \\
\hline & rVVC & $\varnothing$. & अपे? & $(7.16)$ \\
\hline & & $-\mathrm{r}-$ & & $(7.16)$ \\
\hline & CVr.CVr & $\mathrm{CVr}$ & & 1.9 \\
\hline & $-r$ & $-r$ & & 7.4 \\
\hline \multirow[t]{2}{*}{ RV.CV } & RV.CV & YV.CV & 7.1 & 1.1 \\
\hline & & $\mathrm{yVC}$ & 6.1 & 7.6 \\
\hline \multirow[t]{7}{*}{$-\mathbf{R}-$} & $-\mathbf{R}-$ & $-\gamma-$ & 8.7 & 2.5 \\
\hline & & $-[\mathrm{Y}]-$ & 1.8 & \\
\hline & & $\gamma^{-}$ & $7.4,8.2$ & $7.12,8.21$ \\
\hline & & $-\gamma$ & 8.20 & $8.4,8.15$ \\
\hline & & $-\mathrm{R}$ - & 4.14 & 7.10 \\
\hline & & $\mathrm{R}-$ & 6.3 & \\
\hline & & $-\mathrm{r}[$ & 8.23 & \\
\hline \multirow[t]{4}{*}{$-\mathbf{R}$} & $-\mathbf{R}$ & -8 & $4.7,8.12,8.19$ & 8.8 \\
\hline & & -R & $3.2,3.6$ & \\
\hline & & $-[R]$ & 4.13 & \\
\hline & & $-[R]$ & 7.2 & \\
\hline
\end{tabular}


Table 8.11 Results of the evaluation of Hayes' basic vocabulary comparisons

\begin{tabular}{lcccc}
\hline & Probable & Possible & Weak & Rejected \\
\hline A. PAA-PAN & 9 & 9 & 2 & 12 \\
B. PAA-PMP & 3 & 5 & 9 & 8 \\
C. PEAA-PAN & 3 & 5 & 0 & 5 \\
D. PEAA-PMP & 2 & 3 & 1 & 7 \\
Totals & 17 & 22 & 12 & 24 \\
\hline
\end{tabular}

The Appendix to this chapter provides my evaluation of what I consider to be the potentially corresponding forms among the Hayes' list of basic vocabulary. Table 8.11 summarises the results.

\section{The morphosyntactic evidence for Austric}

Ross (2000: 447), in his careful review of my most recent paper on the subject (Reid 1999), wondered whether I was no longer satisfied that the morphosyntactic evidence I had cited in my earlier paper was still viable as evidence. In that I was presenting 'new evidence for the hypothesis' I did not think it was necessary to restate the old evidence. ${ }^{1}$ However, for those who may not be familiar with the earlier work in this area, I will restate it here.

Evidence given in my 1994 paper includes:

1 The AA causatives *pa- $/<$ ap $>$ and *ka- are considered to correspond to AN causatives *pa-, *ka- and *paka-.

2 The AA agentives * <um $>$ and *ma- $/<$ am $>$ are considered to correspond to AN agentives *mu- $/<$ um $>$ and *maRa-

3 The AA instrumentals *<an $>,<$ in $>$ are considered to correspond to EF instrumental *paN-, and AN nominalising affix *ni- $/<$ in $>$, respectively.

4 The AA objective *-a is considered to correspond to AN objective *-a.

5 Evidence from Sora, Khasi, Nancowry and Car Nicobarese suggest a PAA attributive linker *(n)a corresponding to the AN 'ligature'*(n)a

6 The Nicobarese determiners marking case of NPs Pin, Pan, nun, etc., appear to have developed by the same well-known grammatical processes that have brought about the nasal final determiners in many AN languages, that is, by the fusion of a reduced form of the ligature *na. What is important here is that the Malayic languages reflect a PMP (my use of the term, not Blust's) innovation *na $>$ *na, hence Tag. ang, nang Kawi ang, Malay yang, etc., so that the Nicobarese forms could not have been borrowed from sailors speaking a Malayic language.

7 Evidence from Nicobarese, Old Khmer, Khmu and Mal suggest a PAA *ta 'locative' preposition corresponding to PAN 'locative preposition, demonstrative' *ta.

8 Car Nicobarese $2 i$ 'locative preposition' corresponds to PAN *i 'locative preposition'. Note that although reflexes of this preposition are found all the way to Proto-Polynesian, the Proto-Malayic locative preposition is 
reconstructed as *di (Adelaar 1992), and is a reflex of PAN *di 'locative preposition, demonstrative', so if borrowed, the Nicobarese locative preposition could not have come from sailors speaking a Malayic language.

Ross (2000: 446-7) neatly summarises the morphosyntactic evidence that I had presented in my $8 \mathrm{ICAL}$ paper as follows:

9 The Nicobarese causative verbal infix $\langle u m\rangle$ is taken to be cognate with PAN infix $*<$ um $>$ that marked a verb as an unergative intransitive and formed deverbal nouns expressing non-agentive causers (e.g. Bontok $s<u m>$ akit 'that which makes [someone] sick'). (Refer also Schmidt 1916.)

10 PAN and PAA are both taken to have been ergative with a contrast between nominative and genitive pronouns, the genitive denoting both possessor and transitive agent.

11 The PAN and PAA first-person singular pronouns appear to be cognate.

12 Nominative pronouns in both PAN and PAA are taken to have been prefixed with *a-.

13 Ruc, a conservative language of the Vietic branch of AA, has a dative prefix $p a$ - that appears to be cognate with PAN *pa 'go'.

14 A non-proximal demonstrative *en is reflected in both AA and AN languages.

Several arguments have been raised in recent years in an attempt to find alternatives to the morphological comparisons cited earlier.

Borrowing is the primary explanation that has been proposed. Sagart (email comm. 2001) states, 'I have come to the conclusion that the "accidental/involuntary action" prefix t $a$ - in AA languages of Vietnam: Pacoh, Chrau, Katu and Bahnar, is borrowed from Chamic. So this argues that transmission of morphology from AN to AA is possible'.

There is little question that morphological processes can be borrowed between languages of different families. Whether or not it is possible for 'morphemes', that is, meaningful phonological units which constitute part of a word, to be borrowed without their host words also being borrowed is a matter still open for discussion. But whatever the answer to that question, the morpheme $t a$-itself has not been proposed as evidence for Austric, and what is more important, the morphemes that have been proposed have such an extensive distribution, within the AA family, that no reasonable explanation can be given for either the time of borrowing or the possible source language.

The possibility of borrowing seems likely in the case of the strong Nicobarese morphosyntactic similarities with AN, where it is assumed early AN sailors may have made frequent landfall, perhaps in some cases staying, intermarrying and influencing the local language. But there remain two strong barriers to the acceptance of this position. One is that several of the proposed comparisons between Nicobarese languages and AN are not limited to Nicobarese, but are found across wide areas of the AA family. Comparisons in some cases (especially $<u m>$ and $<$ in $>$, however, are clearest with Nicobarese because other EAA languages have either lost the form (in the 
case of verbal suffixes) or modified them as the result of the strong areal influence of Chinese. Moreover it is clear that if Nicobarese borrowed the suspect forms, it could not have been from a language of the Malayic family, because by ProtoMalayic times the forms had been either lost or changed from the earlier forms that I claim are reflected in Nicobarese (as noted earlier (6) and (8)).

The claim has also been made (Sagart email comm. 2001) that the vowels of the *mu- $/<$ um $>$ and *ni- $<$ in $>$ affixes

are more or less colored by the main consonant, they could be secondary. The vowel is essentially an epenthetic schwa which serves to break the consonant cluster formed by prefixed $m$ - or $n$ - and the root initial, or the root initial plus infixed $-m$ - and $-n-$. In both cases the prefix or infix tends to color the schwa: you get rounding with $m$, and a high-and-somewhat fronted vowel with $n$. It is possible that in each particular word the root initial also plays a role coloring schwa.

Whether or not this may ultimately be the source of the vowels in these affixes, the evidence that we have from Nicobarese, and from AN languages suggests that in these languages at least, the vowels of the affixes were full vowels, and not epenthetic. PAN alone has a number of reconstructions which show $m$ followed and/or preceded by vowels other than $u$ (e.g. *mimah 'drink', *SimaR 'fat, oil', *maCa 'eye', "gemgem 'hold (in fist)', etc.), and $n$ followed and/or preceded by vowels other than $i$ (e.g. *tanek 'cook', *tenem 'sea, saltwater', etc.). Similar data could be drawn from Nicobarese as well as from Munda languages, and these are the only places that one could look in AA for evidence to support the thesis.

With respect to my claim that the AA instrumentals $*<$ an $>,<$ in $>$ are considered to correspond to the EF instrumental *paN-, and the AN nominalising affix ${ }^{*} n \mathrm{i}-/<$ in $>$, respectively, the argument has been made that the functions of the AA and $\mathrm{AN}$ affixes are not close enough, the former being 'instrumental' or 'agentive' (Thurgood 1999) while the latter was an 'objective' nominalisation specifying the result of the action of the verb. However, AA data not only shows 'instrumental' nominalisations, but nominalisations of the AN type. Thurgood (1999: 245) quotes Banker as claiming that the Bahnar infix $\langle\partial n\rangle$ sometimes means 'the result of a verbal action', so that bat 'to make a dam', becomes $b<\partial n>\hat{a} t$ 'a dam'. It is probable that the agentive function of the affix was also present in PAN, given the form ${ }^{*} \mathrm{C}<\mathrm{in}>$ aqi 'guts', which can only be interpreted as meaning 'that which produces "Caqi "faeces" ". That the same affix can be reconstructed with these two apparently quite different functions should not be surprising, given the fact that forms such as $b<\partial n>\hat{a} t$ 'a dam' are potentially ambiguous between 'the object that is the result of damming', and 'the object that dams'.

One of the facts which is of high value in supporting the morphological comparisons between AA and AN languages, is not simply the forms and their functions, but also the apparently unique phonological process (not, as far as I know, reported anywhere else in the world), whereby the consonant of the affix and the initial consonant 
of the root metathesise, producing alternation historically not only between the $*^{*}$ mu- $/<$ um $>$ and *ni- $/<$ in $>$ affixes, but also between the *ma- $/<$ am $>$ and *pa- $<$ ap $>$ affixes, with varying distributions in both families, and clearly with the original metathesis (producing infixes) reversible, so that in some daughter languages the prefixal forms reappear either alone, or in alternation with the infix, depending (usually) upon the manner of articulation of the root-initial consonant.

Ross (2000) has questioned the identification of * mu- $/<u m>$ as a causative nominaliser in PAN, suggesting that it is by no means certain that it was a nominaliser at all, thereby questioning its functional association with the corresponding PAA forms. If PAN was anything like most present-day AN languages, whether or not it functioned primarily as a nominaliser or not, verbs that were formed with it, would also have been zero-derived as nouns, when appearing as the heads of noun phrases, just as the Bontok form $s<u m>a k i t$ can mean either 'to make one sick' when occurring as a verb, or 'the thing that makes one sick', when occurring as a noun.

He further questions my identification of *a- as a nominative marker in PAA and PAN with the observation that in Taoih, this prefix is found not only on subject pronouns, but on dative pronouns as well. Of course, if PAA was ergative, as I claim, what is today a dative pronoun in an accusative language (as Taoih probably is) would have been the grammatical subject of a transitive sentence at an earlier stage and would have been marked as nominative, as it is in AN languages that are ergative, for example, Bontok,

$\begin{array}{llll}\text { Agtam } & \text { sak-en } & \text { si } & \text { itab } \\ \text { give.Gen.2s } & \text { Nom.1s } & \text { of } & \text { beans } \\ \text { Give me some beans. } & & \end{array}$

To me this is further evidence in support of the ergative nature of PAA. Compare also the ?an subject marking of some NPs in Nicobarese. Ross questions my claim that the initial *a- of the PAN nominative pronouns *aku, *aken, *aten and *amen was the original nominative marking component of these forms, by claiming that genitive forms such as Seediq $n$-aku, Pazeh n-aki, Thao n-ak,n-am, Amis $n$-ako, etc., showed that even in PAN the *a- was part of the pronominal root and could occur as a genitive. The hyphens in these forms represent Ross' analysis of the forms, as having an intial $n$-genitive marker. I claim however that such forms are better analysed as: Seediq $n a-k u$, Pazeh $n a-k i$, Thao $n a-k, n a-m$, Amis na-ko, etc., an analysis which is more consistent with general patterns of genitive noun formation in a wide range of AN languages, including Talubin Bontok in which nak 'my', nam 'your' have independently developed from a combination of na 'the, non-referential noun' plus genitive pronominal endings.

\section{Conclusion}

Ross claims that in order to be convinced of the validity of Austric, he would need either a substantial quantity of regularly corresponding cognates, or a seemingly cognate paradigm of grammatical morphemes. It would be great if we were able 
to provide either one or the other, but at the time-depths we are looking at, and the imperfect state of our knowledge of AA languages, and the extremely limited amount of reconstruction that has been done in the family (compared at least to the AN side), what I have presented in this chapter is at present the best we can do. I believe that the number of apparent cognates cited here between PAA (and PEAA) and PAN (and PMP) from the area of basic vocabulary, come close to providing such a convincing body. The hope of providing seeming cognate paradigms of grammatical morphemes comes closest with the sets of what were probably originally demonstrative nouns, but which in both families have grammaticised into a wide range of determiners, ligatures, prepositions and the like. There is no question that the range of forms is there, including *a, *ta and $*^{n a}$, with corresponding functions on both sides of the family. It is unlikely, however, that it will be possible to find any paradigm of verbal morphology (which I suspect is what Ross is looking for), because I don't think there was much of this in early AN. The paradigm of so-called 'focus' morphology, even if it was present in PAN (which I think is doubtful), is clearly a catch-bag of prefixes, infixes and suffixes which must have existed in pre-AN times as probably nominalising affixes, but never in any sense constituting a paradigm.

The evidence then is not as convincing as one would like, but as Diffloth said in 1994, the evidence 'is undoubtedly there', and I believe it is considerably stronger now, than it was then. The evidence is for a genetic relationship, but is it evident that the families in question descended from a common immediate ancestor, Proto-Austric? With the accumulation of evidence presented by Sagart in this volume and elsewhere, that AN can also be shown to be genetically related to the Sino-Tibetan family of languages, and his claim (Sagart p.c.) that some of the lexical items and affixes claimed to be shared by AN and AA are found also in Sino-Tibetan languages, the possibility exists that the relationship between AA and $\mathrm{AN}$ is more remote than earlier considered. The concept of 'Austric' as a language family may eventually need to be abandoned in favour of a wider language family which can be shown to include both AN and AA language families, but not necessarily as sisters of a common ancestor.

\section{Appendix}

\section{Evaluation of potentially corresponding forms among the Hayes list of basic vocabulary}

A. PAA-PAN

1. Probable

ashes

PAA *qabuh

PAN *qabu

Pacoh abóh, Chrau vuh 'ashes',
Bonda $b u$ ? 'to smoke'

Comments: Accepted by Diffloth as possible (1994: 313). Restricted to Katuic (Pacoh) and Bahnaric (Stieng and Sre), and a Munda cognate with questionable semantics. Probably not borrowed. 


\section{$\operatorname{dog}$}

PAA *cu(q)

PAN *asu

Bonda guso?, PW *so?, VN chó 'dog'

Comments: Accepted by Diffloth as probable (1994: 313).

\section{snake}

PAA *[su](1)aR

PAN *SulaR

Kharia lur, Sora lo?or, (CF lor) 'a kind of snake', Bahnar 'bih tep-lar 'a very small snake that is extremely poisonous' Comments: Possible final syllable reflexes in Munda and Bahnar.

\section{belly}

PAA *ta?al, *ti?al

PAN *tiaN

Sora ta?al 'spleen', Thavung kha?al 'belly, stomach, abdomen', Pacoh acheal 'heart'

Comments: Final syllables match from Munda through the Muong and Katuic comparisons. Semantics are acceptable, and PAA *1 corresponds to PAN $* \mathrm{~N}$ in several cases.

\section{eye}

PAA *mə(n)ta(q)

PAN *maCa

Kharia (V250) mo'd, PVM *mat 'eye', Proto-Plang *hak ${ }^{1}$-kita? ${ }^{1}$

'eyebrow'

Comments: Accepted by Diffloth as probable (1994: 317). Note ProtoPlang (Waic) *hak ${ }^{1}$-kita? ' 'eyebrow' Lit. hair-eye, which supports the final PAA syllable.

\section{father}

PAA *(qa)ma(ma)

PAN *t-ama

Santali mama 'maternal uncle', Katu ama, Pacoh a-ám 'father', Bahnar ma 'younger brother of father or mother'
Comments: Widely distributed with appropriate phonology and semantics, but suspect as a possible nursery word.

\section{mother}

PAA *(na)na

PAN *t-ina

Kharia nana 'elder sister', Bonda tuna 'younger sister (addressed by a brother), wife's younger brother's wife', Sedang na 'older sister, cousin' Comments: Widely distributed with appropriate phonology and semantics, but suspect as a possible nursery word.

\section{rotten}

PAA *yok

PAN *ma-buRuk

Kharia lorog 'to rot, decay', VN ruc 'be rotten', NK phròok 'spoiled'

Comments: Phonologically plausible with possible reflexes in Munda, Monic and Viet-Muong groups.

buy

PAA *po[1]i

PAN *beli

Khasi pli 'change', MUK pál, pánh 'sell', Kharia patay 'fix price, bargain' Comments: The Khasi, Muong and Katuic forms appear to be cognate, and probably correspond to the PAN form.

\section{Possible}

salt

PAA *qa(m)puc(i)

PAN *timus

Pareng b.sut, Kuy pos, Jehai mpoj 'salt'

Comments: Possible cognates in Munda, Pearic, and Aslian.

smoke

PAA *(m)pal

PAN *qebel 
Sora poro 'become smoky', mor 'spread as smoke', Stieng pôr 'smoke out of a hollow tree'

Comments: Possible cognates in Munda and Bahnaric.

\section{egg}

PAA *teloR

PAN *qiCeluR

Juang susuter(o), Pacoh tireal, tirōl, PVM *t(o)lur? 'egg'

Comments: The Munda comparison is doubtful. The Katuic and Viet-Muong comparisons seem more secure.

\section{male/man}

PAA *(n)qalay

PAN *ma-RuqaNay

Bonda laïbu? 'male pig', Pacoh alay, Stieng clay 'brother-in-law'

Comments: The Munda, Katuic and Bahnaric forms possibly correspond. If they do, they probably correspond to the PAN form.

rat

$\mathrm{PAA} * \mathrm{~b}[$ əw]

PAN *labaw

Bonda gubu 'a kind of rat', Riang (Black) kăbu ${ }^{1}$ 'rat, mouse', Mah Meri (Bes. K.L., R33) kanē'rěbu 'mouse' Comments: The Munda, Bahnaric and Aslian forms seem to be cognate. The final syllable is a possible comparison with the PAN form. None of the AA initial syllables correspond with the PAN initial syllable.

\section{head}

PAA *(n)qolu(q)

PAN *qulu

Bahnar (PB) kŏl, Jeh kãl, Mal kliq 'head'

Comments: Reasonable phonological correspondences between Bahnaric and Aslian, possibly corresponding with the PAN form.

shoulder

PAA *(ba)Ra(q)

PAN *qabaRa

Kharia taran, Theng blah 'shoulder', Khasi ta-bla 'shoulder piece of animal' Comments: The Khmuic and Khasi comparisons appear good, and probably correspond with the PAN form, but the Munda term is questionable.

hold (in fist)

PAA *(n)kam

PAN *gemgem

Kensiu cəkam, VN (*gəm>) câm 'hold', Sora kum-si: 'hold in one's fist, hold a handful'

Comments: The Aslian, Vietic and Sora are possible cognates, and if so, probably correspond well with the PAN form.

yawn

PAA *(can)qap

PAN *ma-Suab

Santali (V68) ango'b, PM *sy?aap, VN ngáp 'yawn'

Comments: The Munda, Vietic and Mon forms are possible cognates. They possibly correspond to the PAN form.

\section{Weak}

\section{meat/flesh}

PAA *c $(i$, ə) $c i$

PAN *Sesi/isi

Sora sissid, VN ("ñśic >) thit 'flesh, meat', PM *sac 'fruit, nut, berry, acorn, pod'

Comments: Insecure phonological correspondences.

swim

PAA *la(n)[?]oy

PAN *Nayuy 
Mundari (K519) oiyar, Ruc, loy, Riang Lang_hyy 'swim'

Comments: The Munda form is probably not cognate with the eastern forms. The Palaungic and Ruc forms are likewise doubtful comparisons.

\section{Rejected}

\section{breast}

PAA *n[s]uq

PAN *susu

Bonda da?tu kuri, PW "tis 'breast', Semai ntoh 'chest'

Comments: Phonological correspondences don't work.

\section{neck}

PAA *(n)qe[R]

PAN *liqe R

MUK kel 'neck', Pacoh cŏl 'wear around neck', Sengoi kelkeil 'ankle, wrist'

Comments: The Muong form possibly corresponds with the PAN form, but the Katuic and Aslian forms are semantically doubtful.

\section{female/woman}

PAA *a[x]i, *bu[x]i, *mpa[x]i,

PAN *bahi

Pacoh $a-i$ 'mother', Kharia (K349) bui 'girl', Mon imbay 'elder brother's wife, husband's elder sister'

Comments: Unconvincing phonological and semantic correspondence.

\section{big}

PAA *(can)yaya (q)

PAN *ma-Raya

PW *ra 'big', Theng ya? 'far', Sora (V40) sana: $j$-an 'be at a distance'

Comments: The Proto-Wa form is possibly cognate, but the semantics and phonology of the Khmuic and Munda forms are unconvincing.

\section{shy/ashamed}

PAA *g(i,a)haq(i)

PAN *ma-Siaq

Bonda ğak' 'shame', PW *[gac] 'ashamed, shy', Stieng haas 'feel ashamed, bashful'

Comments: Phonological correspondence with the PAN form is unlikely.

\section{bite}

PAA *(n)yat(i)

PAN *kaRat

Sora (V334) gad 'cut', raj 'cut into small pieces as wood', PM *rac 'cut with a sickle, reap', Katu karóóch 'cut kernels off'

Comments: Phonology may be possible, but semantics unlikely.

\section{dream}

PAA *(m)pe(qi)

PAN *Sepi

Chrau v̌q , Katu bâch 'lie down, sleep', Sora mimid 'sleepy'

Comments: Phonological correspondences don't work.

\section{hunt}

PAA *(n) [r]op, *(c,s)[r]op

PAN *qaNup

Jeh rŭp 'catch, seize', Khasi kynrup 'pounce upon, seize', Bonda sop' 'hold, catch', Stieng choop 'hunt'

Comments: Only the rhyme works.

\section{sleep}

PAA *(n)zoy

PAN *tuduR

Pacoh chur 'sleepy or sad eyes', Birhor (V111) durum 'to sleep', Khmu' hmdir 'to snore'

Comments: Unlikely semantics and phonology. 
steal

PAA *kalaw *kumlaw

PAN *Cakaw

Nicobar kalo:-hayo 'steal', Mundari (V242) kumru, Santali kombro 'thief, theft, steal'

Comments: Phonological correspondences unlikely.

suck

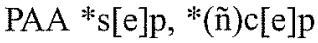

PAN *sepsep

VN top 'sip', Mundari (V354) si'b 'to smoke', Pacoh dyép 'suck'

Comments: Phonological correspondences unlikely.

vomit

PAA *taq

PAN *utaq

PM *taa? 'vomit', Bonda ta?mi 'sneeze',

Semelai tahtoh 'to spit'

Comments: Unlikely semantics.

B. PEAA-PAN

1. Probable

lake

PEAA \{*klow (Hayes)

PAN *danaw

Chrau tanlô 'lake, pond'

Comments: Hayes notes that the lateral in the Chrau reflex he cites (tanlô 'lake') suggests that the form could not be a borrowing from Chamic.

\section{head louse}

PEAA **k[o]t(i)

PAN *kuCu

Katu koót 'lice, fleas, bugs', Khmer sarikœc 'bedbug, flea', Chrau sicăch 'tick'

Comments: With apparent cognates in

Katuic, Bahnaric and Khmer, this is an attractive set to correspond with the PAN form. afraid

PEAA **(n)k[o]t

PAN * ma-takut

Jeh kokilat 'detest, hate', Khmer kot 'hold in awe', Mon takuit 'take fright' Comments: With apparent cognates in Aslian, and both Mon and Khmer, the phonology looks reasonable and the semantics plausible.

\section{Possible}

\section{wood}

PEAA **k(i,a)hi(uq)

PAN *kaSiw

PW *kho?'tree', PM *chuu? 'tree, wood', Semai jahul? 'tree'

Comments: An interesting set of forms which look as though they may well be cognate but the phonological correspondences are uncertain.

\section{fruit}

PEAA ** $(\mathrm{m}) \mathrm{b}[\mathrm{o} \mathrm{a} a] \mathrm{q}$

PAN *buaq

Kensiu kabə?, Sabum kamว? 'fruit', Stieng moq 'type of small fruit'

Comments: The Aslian and Bahnaric appear to be cognate, and there is the possibility that they correspond also with the PAN form.

\section{new}

PEAA **qi[R]u(q)

PAN *ma-baqeRu

PW *cro? 'new', Bahnar chrêu strange' Comments: The Waic and Bahnaric forms seem to be cognate. At least their final syllable may correspond to the PAN form.

\section{burn}

PEAA **[?]us(i), **t[u] nus

PAN *CuNuh

Katu pa-óh 'cook', Khmer 'us 'firewood', Bahnar tonuh 'hearth' 
Comments: The Bahnar form is suspiciously similar to the PAN form. But the form is not reconstructed for ProtoChamic and is probably not a borrowing. Other AA languages show a lateral corresponding to $\mathrm{PAN} * \mathrm{~N}$.

cut (wood)

PEAA **tayaq, **tayak

PAN *taRaq

Rengao chră 'split, divide, crack open', Sre trac 'shave', Pacoh tréq 'chop'

Comments: The Bahnaric forms may well be cognate with Pacoh. They may correspond to the PAN form.

\section{Weak}

rain

PEAA **ka[ñj]al

PAN *quzaN

Brou cuyal, Old Mon kyāl, Khmer khya'l 'wind'

Comments: Although the AA forms are probably cognate, only the rhyme appears to correspond with the PAN form.

stone

PEAA **tamuq (Hayes); PMK *təm(o:)? (Diffloth)

PAN *batu

Khasi *máw, PW *smo?, PM *tmoo? 'stone'

Comments: Diffloth (318) notes this as a possible correspondence. The evidence is weak.

\section{burn}

PEAA **[?]us(i), **t[u]nus

PAN *CuNuh

Katu $p a-o ́ h$ 'cook', Khmer 'us 'firewood', Bahnar tonuh 'hearth'

Comments: The Bahnar form is suspiciously similar to the PAN form. But the form is not reconstructed for Proto-Chamic and is probably not a borrowing. Other AA languages show a lateral corresponding to $\mathrm{PAN}{ }^{*} \mathrm{~N}$.

cut (wood)

PEAA **tak, ***tek

PAN *tektek

Katu ntaak 'chop', Pacoh tich 'chop firewood', Rengao kotěk 'snap, break, cut skin'

Comments: The consonants appear to correspond, but the forms are probably onomatopoetic.

\section{Rejected}

\section{moon}

PEAA **b(i,a)!al

PAN *bulaN

Katu baraal 'pale', Bateg Deg

baycl ( $\left.{ }^{*} \mathrm{r}>\mathrm{y}\right)$ 'white', Bahnar

monhal 'very bright light or

sunshine'

Comments: Unlikely semantics.

sand

PEAA **lay

PAN *qenay

VN lây 'miry, swampy, marshy', Bahnar lai 'mound of dirt', Nyah Kur $1 \varepsilon \varepsilon$, rè 'ore, mineral'

Comments: The may phonology be possible, but the semantics are unlikely (none means 'sand').

\section{back}

PEAA **kko[d(i)]

PAN *likud

Boriwen kiat 'back', Halang

kuyqq 'small of the back of the head', Jeh kung kuyq 'back of head'

Comments: The Bahnaric are probably not cognate with Boriwen, nor with the PAN form.

\section{liver}

PEAA *kp[a]le(q)

PAN *qaCay 
Mon pli 'spleen', PVM *ple?,

Sengoi pele 'fruit'

Comments: The AA forms may be cognate, but they certainly don't seem to relate to the PAN form, either in phonology or semantics.

\section{old (people)}

PEAA **ti(n)qas(i)

PAN * ma-tuqaS

Khmer cā's, Pearic čchu:s 'old', Katu takóh 'grown'

Comments: The phonological correspondences between AA and the PAN form don't work.

\section{choose}

PEAA **(u)laqi

PAN *piliq

PW *ras, Pacoh rôih, Semai (Serau, C120A) chenlas 'choose'

Comments: Impossible phonology.

see

$\operatorname{PEAA}\left({ }^{* *}\right)(\mathrm{s})[\mathrm{R}] \mathrm{ai}$

PAN *kita

Pacoh lây, Chrau sây, VN thây 'see'

Comments: Impossible phonology.

sew

PEAA **(n)qiq

PAN *taSiq

Pacoh ěh, Katu jih, Sengoi

ceik 'sew'

Comments: Impossible phonology.

\section{PAA-PMP \\ 1. Probable}

dust

PAA *buk

PMP *qabuk

Bonda tubok'/tubuk' 'earth', Chrau vŏq 'mud', Mon khabuik 'fine powder or dust'
Comments: Reasonable semantics, and good (final syllable) correspondences from Munda through Bahnaric and Mon.

roof/thatch

PAA *(n)təp

PMP *qatep

Khasi tap 'to cover', Mundari (V3)

$d a ' b$ 'cover a roof, thatch', Palaung $d \check{\Lambda} p$ 'to cover, thatch'

Comments: Good phonological and semantic correspondence across AA, with regular correspondences to the PMP form.

split

PAA *(ba)!aq(i)

PMP *belaq

Katu blah 'split', Kharia (V304)

la'j 'slice', Khmer -la's 'separate, detach'

Comments: The Katuic form is cognate with reconstructed forms in PMong *blah, as well as in three branches of Bahnaric (Thurgood 1999: 284) so is probably not a Chamic borrowing.

\section{Possible}

fog/mist

PAA *(m)put

PMP *kabut

Sora (V384) umod-an 'fog, mist', Khmu' (hm)punt 'clouds, fog'

Comments: Restricted distribution to Munda and Khmuic, but semantics are reasonable, and phonological correspondences possible.

\section{spouse}

PAA *saw[a]

PMP * qasawa

Kharia (K535) sou 'husband', Katu sasaau 'father's cousins, sister's husband, father's sister's children', ProtoSemai *bnsaaw 'wife's elder brother' 
Comments: The phonological comparison is attractive, and the semantics possible.

\section{fall}

PAA *t(a,u)(m)puq

PMP *nabuq (?)

Mundari (K149b) tombo? 'fall forwards', Katu tampoh 'drop', Bahnar puh 'slip, fall into a hole'

Comments: The AA forms appear to be cognate, and possibly relate to the PMP form.

\section{stab}

PAA *(n) $\operatorname{cuk}(\mathrm{i})$

PMP *suksuk

Sora suj, VN choc 'pierce', Sengoi cok 'stab, pierce'

Comments: Possibly cognate.

\section{throw}

PAA *da(q)

PMP *tudaq

Santali (V173) lebda 'throw', Khasi $p d a$ 'throw to the farthest distance possible', Chrau randăh 'throw down'

Comments: The final syllable of the AA forms could correspond to the PMP form.

\section{Rejected}

\section{child}

PAA *(n)qu?an[ak]

PMP *anak

Santali (V205) hon 'son, child', PM *koon 'child, offspring, young (animals)', Mintil Rawa? 'child'

Comments: The Aslian form would be good were it not for the medial consonant that doesn't correspond. The other forms don't correspond at all to the PMP form.

\section{cold}

PAA *(z)!en

PMP *ma-dindin

Kharia (K208) rayga, VN

lanh 'cold', Khmer sren 'to cool'

Comments: Phonological correspondence lacking.

\section{breathe}

PAA *r(a, u)wa(i)

PMP *mañawa

Mundari (K537) rowa, Sengoi ruai 'soul, spirit', Pocoh rvai 'soul'

Comments: Phonological correspondence uncertain.

\section{grow}

PAA *m[b]uk

PMP *tu(m)buk

Kharia (V286) mu? 'come out', Muk moc 'grow, come up', OM mok 'appear'

Comments: The Kharia and Old Mon forms appear to be cognate, but the semantics of the Muk form is only questionably related. Insufficient evidence to establish a correspondence with the PMP form.

\section{split}

PAA *(ba)!aq(i)

PMP *belaq

Katu blah 'split', Kharia (V304)

la'j 'slice', Khmer -la's 'separate, detach'

Comments: The Katuic form is cognate with reconstructed forms in PMong *blah, as well as in three branches of Bahnaric (Thurgood 1999: 284) so is probably not a Chamic borrowing.

\section{PEAA-PMP}

\section{Probable}

\section{mouth}

PEAA **(m)paq 
PMP *baqbaq

Pacoh piaq 'mouth, opening, end of river', Mah Meri pak, Sengoi

mpak 'mouth'

Comments: Although corresponding forms are limited to Aslian and Pacoh, it is unlikely because of their shape that they were borrowed from either Malay (in the case of Aslian) or Chamic (in the case of Pacoh). Note PChamic *babah 'mouth' (Thurgood 1999: 283). Probably cognate with the PMP form.

\section{dull/blunt}

PEAA **tu[!]

PMP *pundul

Pacoh tíl múl '(expressive) of blunt end', Bahnar tŭl 'dull, not pointed', NK thuuul 'blunt, not pointed'

Comments: Possibly cognate forms with distribution in Katuic, Bahnaric and Monic branches, appear to correspond well with the PMP form.

\section{Possible}

\section{husband}

PEAA **na(q)

PMP *bana

Thavung $n A A^{2}$ 'mother's younger brothers', Sengoi menah 'parent's younger brother', Bahnar nă 'parent's elder sibling'

Comments: Possible phonology, semantics questionable.

\section{pound}

PEAA **ntuk

PMP *tuktuk

Khmer tuk 'beat, pound', PM

*kndok 'pound (earth)', MUK

(*duk>) tuc 'to chisel'

Comments: Plausible phonological and semantic correspondence but possibly onomatopoetic. tie

PEAA **(n)kət

PMP *hiket

Cua takoot 'tie a knot', VN cột 'tie up, chain', Pearic kho:t 'tie'

Comments: The AA forms are probably cognate, and possibly correspond to the PMP form. The vowel however may be problematic.

\section{Weak}

\section{split}

PEAA **ta[q]

PMP *sitaq

Chrau $t a ̆ h$ 'slit open, cut up', Stieng tah 'disembowel'

Comments: The AA forms clearly cognate, but semantics don't match well with the PMP form.

\section{Rejected}

correct, true

PEAA **kbr, **bənər

PMP *ma-bener

Sengoi bor 'good, fine, beautiful', bernor 'goodness, righteousness, true', Pacoh nnôr 'happy'

Comments: Too restricted distribution in AA, questionable semantics.

\section{dirty}

PEAA **maz

PMP *cemeD (?)

Mon mih 'body dirt', Mintil

kamah 'dirty'

Comments: Too restricted distribution in AA, questionable phonology.

\section{good}

PEAA **i[?]ak, **u[?]aq

PMP *ma-pia

VN uóc 'to desire, wish for, hope for', Khasi kwah [kaw?] 'wish for',

Jeh $w \breve{a}$ [wa?], 'want, like, be fond of, desire' 
Comments: Insufficient phonological correspondence.

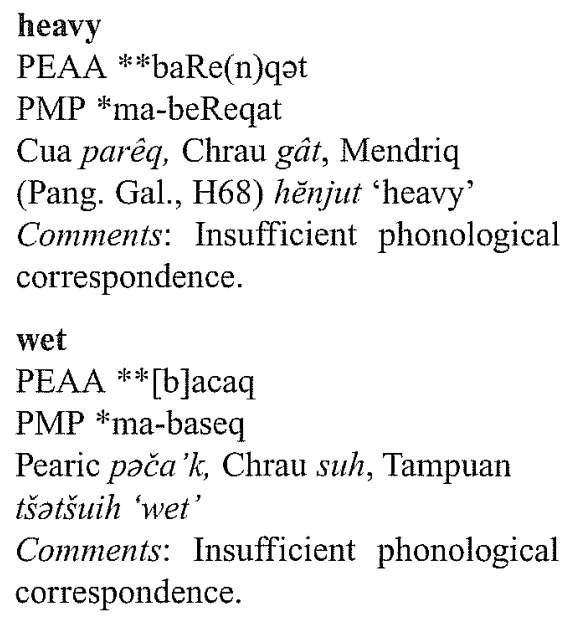

\section{lie down}

PEAA **(i)lap

PMP * qinep

Nha Heun plîp, Thavung kñiip, Bahnar 'nhipp 'close eyes'

Comments: Semantics not close enough.

\section{spit}

PEAA **zaq(i), **ñjaqi

PMP *luzaq

Bahnar kosoh, OM ksas 'spit', Khmer khjä'k 'spit out'

Comments: Insufficient phonological correspondence.

\section{Abbreviations}

$\begin{array}{ll}\text { AA } & \text { Austro-Asiatic } \\ \text { AN } & \text { Austronesian } \\ \text { EAA } & \text { Eastern Austro-Asiatic } \\ \text { PA } & \text { Proto-Austric } \\ \text { PAA } & \text { Proto-Austro-Asiatic } \\ \text { PAN } & \text { Proto-Austronesian } \\ \text { PEAA } & \text { Proto-Eastern Austro-Asiatic } \\ \text { PMP } & \text { Proto-Malayo-polynesian }\end{array}$

\section{Note}

1 Ross also faulted me for including a reference to Hayes' basic vocabulary reconstructions, but not providing any examples. At the time when my paper was presented (at 8ICAL), Hayes' paper was not yet published, and I had been given a pre-publication version of it, with the promise that I could refer to it but not cite any of his data. In addition, I had not at that time had the opportunity of evaluating the quality of his reconstructions.

\section{Bibliography}

Adelaar, K.A. (1992) Proto-Malayic: The Reconstruction of its Phonology and Parts of its Morphology and Lexicon, Canberra: Pacific Linguistics C-119.

Blust, R.A. (1993) 'Central and Central-Eastern Malayo-Polynesian', Oceanic Linguistics 32, $2: 241-94$.

(1999) 'Subgrouping, circularity and extinction: some issues in Austronesian comparative linguistics', in E. Zeitoun and P.J.-K. Li (eds) Selected Papers from the Eighth International Conference on Austronesian Linguistics, Taipei: Academia Sinica. 
Diffloth, G. (1994) 'The lexical evidence for Austric, so far', Oceanic Linguistics 33: 309-22.

Hayes, L.H. (1997) 'On the track of Austric: Part II. Consonant mutation in early Austroasiatic', Mon-Khmer Studies 27: 13-44.

(1999) 'On the track of Austric: Part III. Basic vocabulary comparison', Mon-Khmer Studies 29: 1-34.

Parkin, R. (1991) A Guide to Austroasiatic Speakers and Their Languages, Oceanic Linguistics Special Publication, no. 23, Honolulu: University of Hawai'i Press.

Reid, L.A. (1994) 'Morphological evidence for Austric', Oceanic Linguistics 33, 2: 323-44.

_ (1999) 'New linguistic evidence for the Austric hypothesis', in E. Zeitoun and P.J.-K. Li (eds) Selected Papers from the Eighth International Conference on Austronesian Linguistics, Taipei: Academia Sinica.

Ross, M.D. (2000) 'Review of E. Zeitoun and P.J.-K. Li (eds) Selected Papers from the Eighth International Conference on Austronesian Linguistics', Oceanic Linguistics 39, 2: $445-56$.

Schmidt, W. (1906) 'Die Mon-Khmer-Völker, ein Bindeglied zwischen Völkern Zentralasiens und Austronesiens', Archiv der Anthropologie, n.s. 5: 59-109.

- (1916) 'Eineges über das Infix $m n$ und dessen stellvertreter $p$ in den austroasiatischen Sprachen', Aufsätze zur Kultur- und Sprachgeschichte, vornehmlich des Orients, Ernst Kuhn zum 70, Breslau: Marcus.

Thurgood, G. (1999) From Ancient Cham to Modem Dialects: Two Thousand Years of Language Contact and Change, Oceanic Linguistics Special Publication No. 28, Honolulu: University of Hawai'i Press. 
First published 2005

by RoutledgeCurzon

2 Park Square, Milton Park, Abingdon, Oxon OX14 4RN

Simultaneously published in the USA and Canada by RoutledgeCurzon

270 Madison Ave, New York, NY 10016

RoutledgeCurzon is an imprint of the Taylor \& Francis Group

(C) 2005 Editorial matter and selection, Laurent Sagart, Roger Blench and Alicia Sanchez-Mazas; individual chapters, the contributors

Typeset in Times New Roman by

Newgen Imaging Systems (P) Ltd, Chennai, India

Printed and bound in Great Britain by

MPG Books Ltd, Bodmin

All rights reserved. No Part of this book may be reprinted or reproduced or utilised in any form or by any electronic, mechanical, or other means, now known or hereafter

invented, including photocopying and recording, or in any information storage or retrieval system, without permission in writing from the publishers.

British Library Cataloguing in Publication Data

A catalogue record for this book is available from the British Library

Library of Congress Cataloging in Publication Data

A catalog record of this book has been requested

ISBN 0-415-32242-1 